\title{
Exotic massive hadrons and ultra-high energy cosmic rays
}

\author{
Ivone F. M. Albuquerque円 \\ Department of Astronomy and Astrophysics, Enrico Fermi Institute \\ The University of Chicago, Chicago, Illinois 60637 \\ Glennys R. Farrarf \\ Department of Physics and Astronomy \\ Rutgers University, Piscataway, New Jersey 08855 \\ Edward W. Kolb \\ NASA/Fermilab Astrophysics Center \\ Fermi National Accelerator Laboratory, Batavia, Illinois 60510, and \\ Department of Astronomy and Astrophysics, Enrico Fermi Institute \\ The University of Chicago, Chicago, Illinois 60637
}

\begin{abstract}
We investigate the proposal that primary cosmic rays of energy above the Greisen-Zatsepin-Kuzmin cutoff are exotic massive strongly interacting particles (uhecrons). We study the properties of air showers produced by uhecrons and find that masses in excess of about $50 \mathrm{GeV}$ are inconsistent with the highest energy event observed. We also estimate that with sufficient statistics a uhecron of mass as low as $10 \mathrm{GeV}$ may be distinguished from a proton.
\end{abstract}

PACS number(s): 96.40, 98.70, 11.30.P

\footnotetext{
${ }^{1}$ Electronic mail: ifreire@mafalda.uchicago.edu

${ }^{2}$ Electronic mail: farrar@farrar.rutgers.edu

${ }^{3}$ Electronic mail: rocky@rigoletto.fnal.gov
} 


\section{Introduction}

About thirty years ago it was pointed out that sufficiently energetic cosmic rays will lose energy scattering with the cosmic microwave background (CMB) or radio wavelength background radiation (RBR). Greisen [1] and Zatsepin and Kuzmin [2] (GZK) noticed that above threshold for pion production a cosmic-ray proton will lose energy by pion photoproduction. Sufficiently energetic nuclei also have a limited range before suffering photodissociation. The mean-free-path for a proton with energy above pion production threshold is less than a dozen Mpc, and the mean-free-path for a nucleus above threshold for photodissociation is even shorter円.

With the assumption that very high energy cosmic rays originate from sources more distant than this, Greisen and Zatsepin and Kuzmin predicted that there should be a maximum observed energy in the cosmic-ray spectrum. This maximum energy, known as the GZK cutoff, is usually taken to be around $5 \times 10^{19} \mathrm{eV}$. Surprisingly, today there are more than 20 events observed with energy in excess of the GZK limit, and 8 events J $^{2}$ above $10^{20} \mathrm{eV}$ 4, 5, 6, 7, 8]. The slope of the energy spectrum appears to change around $10^{19} \mathrm{eV}$ (in fact it appears to harden), possibly indicating a new hard component in the cosmic-ray spectrum above the cutoff.

The most straightforward interpretation of the observation of cosmic rays above the GZK cutoff is that the sources of the cosmic rays must be "local" (within $50 \mathrm{Mpc}$ or so). Since cosmic rays above the GZK cutoff should be largely unaffected by intergalactic and galactic magnetic fields, there should be candidate cosmic accelerators associated with the arrival direction of the UHE cosmic rays.] But there seems to be no unusual

\footnotetext{
${ }^{1}$ However, the original estimates of the infrared and far infrared background have been recently reexamined, leading to a possibility of a larger photodissociation mean free path [3].

${ }^{2}$ This number does not include events from southern hemisphere detectors.

${ }^{3}$ In this paper, ultra-high energy (UHE) will refer to energies near or above the GZK cutoff, which we take as $5 \times 10^{19} \mathrm{eV}$.
} 
astrophysical sources such as active galactic nuclei or quasars within $50 \mathrm{Mpc}$ in the direction of the events above the GZK cutoff. There are hints of unusual sources at cosmological distances (redshift of order unity) associated with the arrival directions of the highest energy cosmic rays [9, 10], but there is no way a UHE nucleon or nucleus could reach us from such distances. This suggests that new physics or astrophysics is required to understand the nature, production, composition, and propagation of such high-energy cosmic rays. This issue has attracted a lot of attention, because even the most conservative explanations involve exciting new physics.

The first possible explanation is that the UHE cosmic rays actually do originate within $50 \mathrm{Mpc}$ from us but we cannot see the accelerator. One might argue that the fact that there are events above the GZK cutoff is evidence that there must be galactic or extragalactic regions where the magnetic fields are far stronger than generally believed, so the arrival direction would not be correlated with the source direction [11]. Or, perhaps the reason we do not observe any vestige of the cosmic accelerator is that the acceleration results from a transient phenomenon such as a cosmic defect [12] or a gamma-ray burst in a relatively nearby galaxy [13. In these scenarios the source could be within $50 \mathrm{Mpc}$ and a primary cosmic-ray hadron could reach us without appreciable energy loss. Note however that the integrated flux reduction factor for a $3.2 \times 10^{20} \mathrm{eV}$ cosmic ray is of order 100 or more at 50 Mpc (c.f., Fig. 2 of Ref. [9]).

A second possibility is that the UHE cosmic rays are not nucleons, and originate from sources at cosmological distances without suffering energy loss while propagating through the radiation backgrounds. Stable non-hadronic primary candidates are electrons, photons, and neutrinos. Electrons are not candidates since their energy loss during propagation is huge. Photons interact with the CMB and RBR and lose energy by $e^{+} e^{-}$ production, resulting in a propagation distance comparable to protons. Furthermore, the highest energy cosmic-ray events are unlikely to be due to photons because photon- 
induced showers do not resemble the observed Fly's Eye shower [14] and the muon content of other events is consistent with expectations for a hadronic rather than photon primary [8]. Neutrinos could reach us from cosmological distance without energy loss, but it was shown that even allowing for new physics, the observed events could not be due to neutrino-induced showers [15]. Even with the meager number of UHE events it seems very likely that the atmospheric shower was induced by a strongly interacting particle.

A third possibility is that the primary is a hadron, as suggested by the properties of the observed air showers, but for some reason it has a longer path length than a normal nucleon. There are two ways this might happen. The first way is if the cross section for interaction with the CMB is smaller. The second way is for the energy threshold for resonant photoproduction interactions to be higher, so that the GZK bound is "postponed" to higher energy. Farrar [17] pointed out that the existence in certain supersymmetric models of a new stable hadron (e.g., $S^{0}$ ) with a mass of several GeV can realize this possibility. Due to its greater mass and its large mass-splitting to its resonances, the threshold energy for resonance pion production is increased compared to a proton. This increases its effective path length and postpones the GZK cutoff to higher energy.

A complete calculation of energy loss due to pion photoproduction, redshift, and $e^{+} e^{-}$ production was done by Chung, Farrar and Kolb 18 (CFK) for $S^{0}$ 's in the mass range around $2 \mathrm{GeV}$. Their analysis demonstrated that the effective range through the CMB of a neutral primary as light as $2 \mathrm{GeV}$, with cross sections typical of a neutron but with a larger gap to the lowest resonance, would be fifteen times more than a nucleon. Although their detailed analysis involved masses of around 2-3 GeV, they pointed out that one might entertain the possibility that the primary is much more massive than the nucleon.

\footnotetext{
${ }^{4}$ Weiler has proposed a hybrid model [16] where UHE neutrinos propagate from cosmological distances, then produce hadrons in collisions with massive neutrinos in the galactic halo. This model seems to require several parameters to be finely tuned.
} 
Indeed, extensions of the standard model often involve new colored particles with masses of order a TeV, which may be stable or long-lived. Since the pion production threshold energy is directly proportional to the mass of the primary, the GZK cutoff energy trivially increases for a massive primary and, in addition, the fractional energy loss per collision is smaller for a more massive primary. Thus, from purely kinematical considerations the path length of the primary increases rapidly as its mass increases. If a cosmic accelerator produces primary protons with energies in excess of $10^{21}$ or so $\mathrm{eV}$, then the accelerated proton could collide with a proton at rest near the accelerator and produce massive, high-energy particles with energy above the GZK cutoff: in the collision of a primary of energy $10^{21} \mathrm{eV}$ with a proton at rest, the invariant energy is $\sqrt{s} \sim 10^{3} \mathrm{TeV}$ !

CFK coined the name "uhecron" to describe a new species of long-lived or stable, electrically neutral hadron which, due to its mass, can provide the answer to the cosmicray conundrum. Such a primary of mass $M$ can have sufficient range through the cosmic backgrounds to reach us from sources at cosmological distances ( $L \sim 1 \mathrm{Gpc})$ if its mass is more than a few $\mathrm{GeV}$, and its lifetime is greater than $10^{6} \mathrm{~s}(\mathrm{M} / 3 \mathrm{GeV})(L / 1 \mathrm{Gpc})$.

But, as CFK pointed out, the fact that the uhecron is hadronic does not guarantee that it will shower in the atmosphere like a nucleon. If the uhecron contains a heavy constituent of mass $M$, the fraction of its momentum which is carried by light degrees of freedom is approximately $\Lambda_{Q C D} / M$. It is energy from the light degrees of freedom that is released in a typical hadronic interaction. Thus, if the uhecron contains a massive constituent, the energy spectrum of the particles produced in a uhecron-nucleon interaction will be very soft. CFK argued that for a sufficiently massive uhecron it should be possible to differentiate between a shower induced by a uhecron and a shower induced by a proton.

\footnotetext{
${ }^{5}$ The reason CFK take the uhecron to be neutral is to avoid its interaction with galactic and extragalactic magnetic field and the consequent energy loss due to pair production and other mechanisms. This may not be an essential requirement, depending on the distance to the accelerator.
} 
The purpose of this paper is to determine the maximum possible mass of a primary consistent with present observations by comparing the shower of a massive uhecron with the development of the highest-energy cosmic-ray shower observed, the $E \sim 3.2 \times 10^{20} \mathrm{eV}$ event observed by the Fly's-Eye detector [7]. On the basis of the comparison, we show that the uhecron must be less massive than about $50 \mathrm{GeV}$ in order to explain the highest energy event.

We also perform a more general comparison of proton-induced and uhecron-induced air showers to find means of discriminating between them. Since the fluctuations in the development of hadronic showers is large, it is impossible to identify reliably the difference between a massive uhecron and a proton on a shower-by-shower basis. But we show that with sufficient statistics a uhecron with mass larger than $10 \mathrm{GeV}$ may be distinguishable from proton showers.

It is important again to restate explicitly the picture of the uhecron we will adopt for this study. We will assume the uhecron consists of a single constituent which accounts for the bulk of the mass of the particle, surrounded by light hadronic degrees of freedom (gluons and/or light quarks). This definition of a uhecron strictly does not encompass the archetypical uhecron, the $S^{0}$ [17, 18] which is a bound state of light quarks and a relatively light gluino $(u d s \tilde{g})$. The momentum of the $S^{0}$ is shared roughly equally by all constituents, so its shower properties are expected to resemble those of a nucleon.] However there are a number of interesting uhecron candidates for which the present definition is appropriate:

1. Raby has proposed a class of low-energy SUSY models in which the gluino is the lightest supersymmetric particle [19]. Presumably the lightest color-singlet state containing the gluino is the $R^{0}$ (a gluino-gluon color singlet). For gluinos more

\footnotetext{
${ }^{6}$ Furthermore, even if the $S^{0}$ momentum were carried by a single constituent, our results (see below) indicate that with a mass of order $2 \mathrm{GeV}$ its shower is not distinguishable from a proton.
} 
massive than the QCD scale, the mass of the $R^{0}$ would be approximately the mass of the gluino, which could be anywhere from a few $\mathrm{GeV}$ to $100 \mathrm{GeV}$ in his model.

2. Extensions of the standard model often include new colored particles of mass in the 1 to $10 \mathrm{TeV}$ range, often stable because of some accidental symmetry or a new conserved quantum number (see [18, 20] for examples). Note that cosmological restrictions require them to be unstable over some mass ranges [21].

3. The uhecron may be a massive magnetic monopole (mass around $10^{10 \pm 1} \mathrm{GeV}$ ), accelerated by galactic magnetic fields to UHE energies [22]. The magnetic monopole would have a small core, but could also be surrounded by a gluon cloud. Since the monopole is accelerated by galactic fields, there need be no striking astrophysical sources in the direction of the incoming UHE cosmic rays. Note, however, that it has been argued that their spectrum and arrival direction disagrees with observation [23].

In the next section we describe the longitudinal development of air showers. After a very brief description of the parameterization of the development of the air shower, we describe the procedure used in the simulation of the phenomenon. We then discuss the modifications necessary to study the development of uhecron-induced showers. In Section 3 we present the results of the uhecron simulations and contrast the development of uhecron showers and proton showers. We next compare the uhecron results to the data for the highest-energy event observed and derive a limit on the mass of the uhecron. We then discuss the kind of limit one might conceivably obtain with a larger data set. Finally, before ending the section, we describe the ground-level particle content of a uhecron shower as might be observed with a ground array of detectors. We then present a short concluding section. 


\section{$2 \quad$ Ultra High Energy Air Showers}

\subsection{Shower development}

The highest energy event was detected [7] by the Fly's Eye collaboration using the atmospheric fluorescence technique [24]. An air fluorescence detector measures the longitudinal profile of the air shower. This profile reflects the development of the cascade generated by the interaction of the primary particle with the atmosphere.

The number of particles in the shower grows until it reaches a maximum $\left(N_{M A X}\right)$ at a certain atmosphere depth $\left(X_{M A X}\right)$. The longitudinal development of the number of particles $N$ as a function of atmospheric depth $X$ (measured in $\mathrm{g} \mathrm{cm}^{-2}$ ) in the shower can be fit by the function [25: :7

$$
\frac{N(X)}{N_{M A X}}=\left(\frac{X-X_{0}}{X_{M A X}-X_{0}}\right)^{\left(X_{M A X}-X\right)(10+0.02 X)^{-1}},
$$

where $X_{0}, N_{M A X}$, and $X_{M A X}$ are fit parameters. The energy of the event is proportional to the integral of $N(X)$, the total number of charged particles in the shower.

In addition to giving the energy of the primary, the longitudinal development of the shower can be used to constrain the nature of the primary. Although there are large fluctuations in $X_{M A X}$ on a shower-to-shower basis, the $X_{M A X}$ distribution for many showers differs depending on whether the primary particle is a proton or a nucleus. The reason for this is that a nucleus showers like an ensemble of lower energy nucleons, so that for a given total primary energy, the mean $X_{M A X}$ for the distribution is larger for a proton shower than for an iron shower, i.e., the proton shower peaks deeper in the atmosphere. However, due to the large fluctuations in $X_{M A X}$ the composition determination has a large uncertainty unless there are a large number of events. Thus, there is no clear indication of the composition for the very highest energy cosmic rays [27].

\footnotetext{
${ }^{7}$ This function is a slightly better fit than the familiar Gaisser-Hillas function [26] and was found empirically 25.
} 
Most of the statistics of UHE events have been gathered by extensive air shower (EAS) arrays rather than with the air fluorescence technique. EAS arrays measure the density of particles as a function of the distance from the core, from which the energy of the primary is inferred. The ratio of muons to the electromagnetic density at some distance from the core of the shower $(600 \mathrm{~m}$ or more) can be used to tell whether the showers were induced by photons or hadrons. Discrimination between nucleons and nuclei is more difficult but feasible with sufficient statistics.

In this work, we simulate the development of showers initiated by a $320 \mathrm{EeV}$ uhecron of various masses between 2.5 and $100 \mathrm{GeV}$. We compare the results of their longitudinal development to the shower observed in the highest energy event. We also estimate the ratio between muon and total charged particle densities at ground level. We find that it is not possible to distinguish a proton from a uhecron on a shower-to-shower basis, unless the uhecron is extremely massive. However, with sufficient statistics it may be possible to discern the difference between the showers of a uhecron of mass greater than about $10 \mathrm{GeV}$ and those of a nucleon.

We emphasize that our goal is to look at differences between the shower development of ultrarelativistic nucleon and uhecron primaries. It is known from new data at lower energies that existing shower simulations are inadequate in several respects. This new data will allow improvements in the modeling of the QCD processes, just as modeling of hadronic collisions relevant for discovering new particle physics at LEP, SLC and the FNAL collider gradually improved with more complete data on conventional processes. Likewise, by the time enough events have been accumulated above the GZK bound to apply the kind of analysis we envision, there will be a vastly larger number of events at energies below the GZK bound, where we can be confident the primaries are dominantly conventional particles. That will allow the understanding of conventional particle showers to be improved to the point that we can have confidence in the modeling of the 
showers due to proton primaries above the GZK bound. Then a deviation due to new particles can be studied. Our purpose below is to estimate the extent of the deviation in showering between proton and uhecron primaries which follows from the difference in the momentum-fraction carried by light consituents in a uhecron as compared to a nucleon. We expect this to be a robust discriminator, very weakly dependent of the details of how the light constitutents produce a shower.

\subsection{Air Shower Simulation}

An air shower simulation requires an event generator to simulate the interaction of each shower particle with an atmospheric nucleus and a simulation of the cascade development. In our simulations we use Aires (AIR Shower Extended Simulations) [28] as the cascade simulator ${ }^{8}$ and Sibyll [31] as the event generator. We modified Aires and Sibyll to include a new particle, the uhecron. The modification to Aires was straightforward: adding a new particle to the cascade development. The modifications to Sibyll were more extensive.

Sibyll uses a combination of a model for low energy hadron-hadron interactions, a model for the "hard" part of the cross section, and a model to go from hadron-hadron to hadron-nucleus interactions. For the initial very high energy interactions at $\sqrt{s}$ up to $800 \mathrm{TeV}$, an extrapolation from data taken in laboratory experiments is required. In Sibyll this is done with the Dual Parton Model augmented by minijet production (see [31] and references therein) as will be briefly described below.

In the low energy regime $\left(\sqrt{s} \simeq 10\right.$ to $20 \mathrm{GeV}$ or $E_{\text {lab }}=50$ to $200 \mathrm{GeV}$ ) the observed hadronic cross section exhibits Feynman scaling. At these energies, hadron-hadron interactions can be represented by the production and fragmentation of two QCD strings (see

\footnotetext{
${ }^{8}$ The cascade development used in Aires is similar to the one used in MOCCA [29]. For a comparison between Aires and MOCCA, see 30].
} 
[31] and references therein). The collision occurs between the incoming hadron (either a baryon or a meson) and nucleons in a target nucleus, which is randomly taken to be oxygen or nitrogen. In the case of a baryon-baryon collision the energy of each baryon is divided between one quark $q$ and one diquark $q q$. Sibyll employs a structure function to describe the fraction $x$ of the baryon energy carried by the quark fragment given by

$$
f_{q}(x)=\frac{(1-x)^{\alpha}}{\left(x^{2}+\mu^{2} / s\right)^{1 / 4}} .
$$

Here $\mu$ is considered as an "effective quark mass" and is taken to be $0.35 \mathrm{GeV}$ and $\alpha$ is taken to be 3.0. The diquark carries the remaining energy of the hadron, aside from that part of the hadron energy going into minijet production.

QCD strings are formed between the quark of the incoming particle and the diquark of the target, and vice-versa. The formation of strings obeys energy and momentum conservation. At the end of this process, hadronization occurs. The energy of each produced particle is generated according to the Lund fragmentation function [31]. At each interaction, "leading particles" are produced. They are the baryons which contain the original diquark from the incoming particle and from the target nucleons. The energy carried by these particles is described by a harder fragmentation function than that for the non-leading particles.

\subsection{Modifications to Sibyll to include a uhecron}

We modify Sibyll as described below to account for the difference between the energy deposition properties of a hadron containing a heavy constituent "clothed" by light quarks and gluons (a uhecron) and the energy deposition of a normal hadron whose momentum is carried only by light constituents. As is well understood in the context of Heavy Quark Effective Theory (HQET) [32], the interaction of the uhecron with matter is dominated by the interaction of its light degrees of freedom (quarks and/or gluons). 
Hadronic total cross sections are determined by the spatial extent of the colored quanta. By the uncertainty principle, this is greatest for the lightest degrees of freedom, so we take as our canonical choice for the uhecron-nucleon cross section $\sigma_{U N}=\sigma_{\pi N}$, since the light degrees of freedom are light quarks, antiquarks, and gluons for nucleon, mesons and uhecron. However we also consider explore the effect of taking $\sigma_{U N}=1 / 10 \sigma_{\pi N}$. This lower cross section is motivated by lattice QCD studies which show that the radii of glueballs tend to be two to four times smaller than the radii of ordinary mesons. The reduced size of a hadron containing a valence color-octet particle is understood as being due to its larger color charge and thus stronger confining potential. Thus, if the uhecron contains a heavy color-octet constituent such as a gluino, the lower cross section may be appropriate.

In an ultrarelativistic uhecron, as in a B meson, the heavy constituent (mass $m_{Q}$ ) has most of the momentum of the uhecron and the light degrees of freedom are left with only a small fraction. In our modification of Sibyll, we use for the uhecron the standard Peterson function [33] to describe the fraction of energy $z$ of a heavy hadron carried by its heavy quark $Q$ :

$$
f_{Q}(z)=\frac{1}{z}\left[1-\frac{1}{z}-\frac{\epsilon_{Q}}{1-z}\right]^{-2} .
$$

Here $\epsilon_{Q}$ is proportional to $\Lambda_{\mathrm{QCD}}^{2} / m_{Q}^{2}$. We use this function to replace the hadronic structure function, Eq. (2), and the fragmentation function of a standard hadron. In the fragmentation function the final hadron carries a fraction $z$ of the energy of its parent "quark". This is a hard fragmentation function, and in the limit $m_{Q} \rightarrow \infty$ it approaches a delta function peaked at 1 . We use the Peterson fragmention function since it is in good agreement [33] with data for $b$ quark interactions and has the required qualitative behavior in the limit of extremely heavy constituent.

In the hadron-hadron interaction model employed by Sibyll the quarks that belonged 
to the original baryon undergo a hadronization process in which different probabilities are taken for forming various hadrons [31]. In the uhecron case, the only possibility is for the heavy "quark" $Q$ to hadronize as a uhecron.

Sibyll also includes diffractive dissociation. When diffraction occurs the incoming particle is excited into a higher mass state and then decays if its mass is not large. For larger masses, as in the uhecron case, the excited state does not decay and is instead split, e.g., into a quark and a diquark in the baryon case. These move apart stretching a string between them. The string fragments as in the nondiffractive case. The only modification in the diffractive part of Sibyll was to modify the minimal mass limit of the excited state according to the uhecron mass.

A final modification was related to the "hard" part of the cross section in which the momentum transfer is large. In this case Sibyll models the production of minijets with energies of several $\mathrm{GeV}$. As the uhecron interacts always with low energy because most of the initial energy is carried by the heavy quark that does not interact, we neglect minijet production when the incident particle is a uhecron. Of course, ordinary hadrons are created even in a uhecron-initiated shower and minijets can be produced from the subsequent interactions of these normal hadrons. 


\section{Results of the simulations}

\subsection{Longitudinal shower development}

Assuming a shower energy of $320 \mathrm{EeV}$, in Fig. 1 we show the longitudinal distributions (see Section 2) for protons, $2.5 \mathrm{GeV}$ uhecrons, $10 \mathrm{GeV}$ uhecrons, and $50 \mathrm{GeV}$ uhecrons.[? For each case, the histogram is the single best representation of 500 shower simulations, and the solid line is the best fit to the histogram using Eq. (1).

The first plot shows iron and proton showers for a reference. The other plots in this figure show that $\left\langle X_{M A X}\right\rangle$ increases as the uhecron mass increases. The shower maximum is deeper for a heavier uhecron because the energy loss per uhecron-nucleon collision decreases as the uhecron mass increases. The profile for a typical $2.5 \mathrm{GeV}$ uhecron resembles that of a proton, but $\left\langle X_{M A X}\right\rangle$ is approximately $40 \mathrm{~g} \mathrm{~cm}^{-2}$ deeper and $N_{M A X}$ is lower. The average profile for a $50 \mathrm{GeV}$ uhecron appears very different than the proton. However, it would be hard to distinguish a $50 \mathrm{GeV}$ uhecron from a proton for an individual shower just based on its longitudinal distribution. This is because of the large fluctuations in the the value of $X_{M A X}$ for hadron-initiated showers.

In order to study the $X_{M A X}$ fluctuations, we show in Fig. 22 the $X_{M A X}$ distribution for the 500 showers used in Fig. 1. It is evident that as the uhecron mass increases $\left\langle X_{M A X}\right\rangle$ increases and the distribution becomes broader. This broadening of the distribution makes it difficult for a single event identification of a high-mass uhecron since $X_{M A X}$ fluctuates more when the particle mass is higher. Conversely, given a large number of events, the observed dispersion in $X_{M A X}$ would itself be a useful tool in identifying a uhecron and inferring its mass.

\footnotetext{
${ }^{9}$ Unless otherwise specified the uhecron-nucleon cross section is the same as for a pion-nucleon interaction. We return to the reduced cross section case at the end of this section.
} 

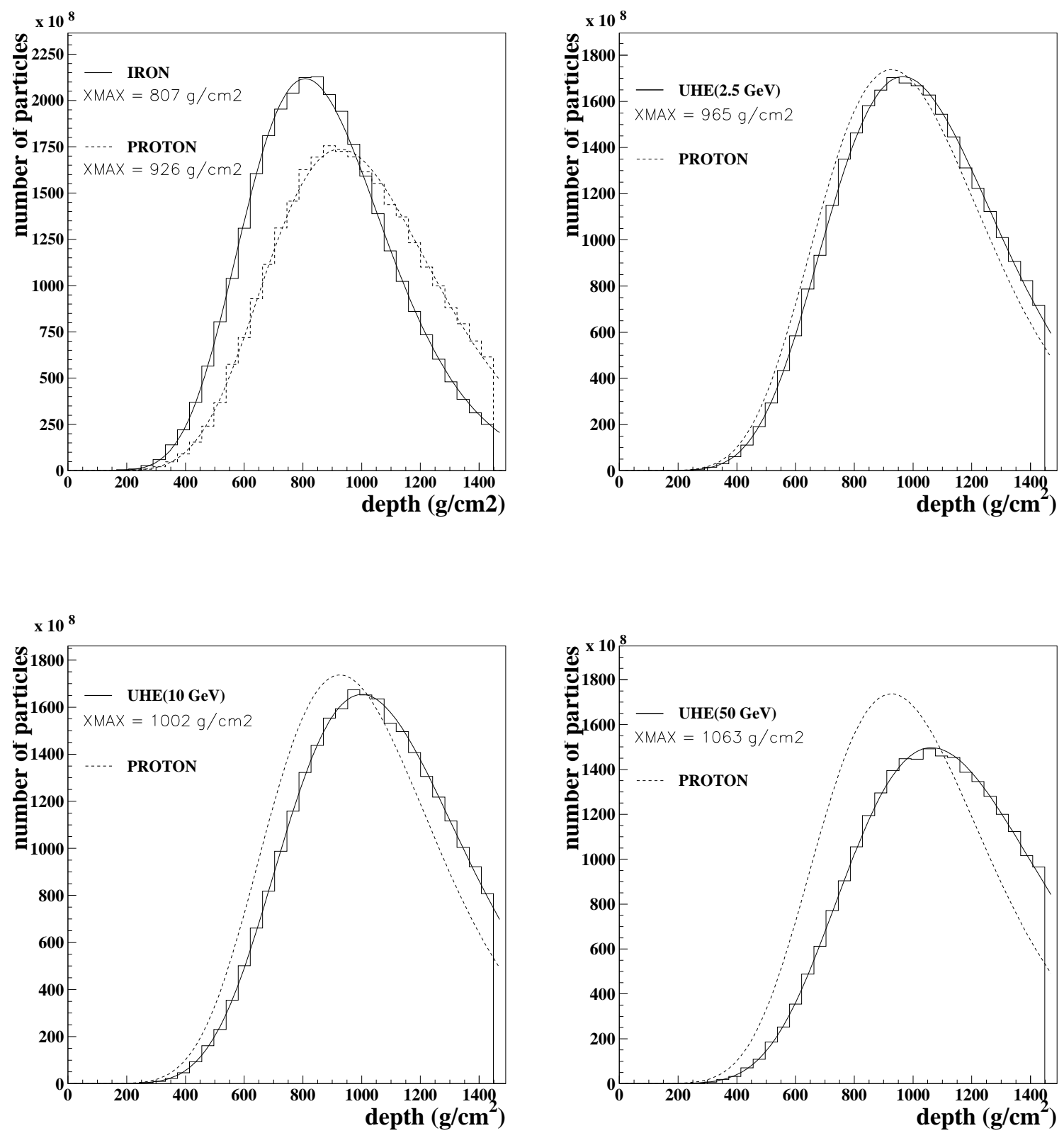

Figure 1: Longitudinal development for $320 \mathrm{EeV}$ showers based on 500 showers for each. All plots are compared with the proton profile: a) iron; b) uhecron $(2.5 \mathrm{GeV})$; c) uhecron $(10 \mathrm{GeV})$; d) uhecron $(50 \mathrm{GeV})$. The fitted $X_{M A X}$ is given for each primary. 

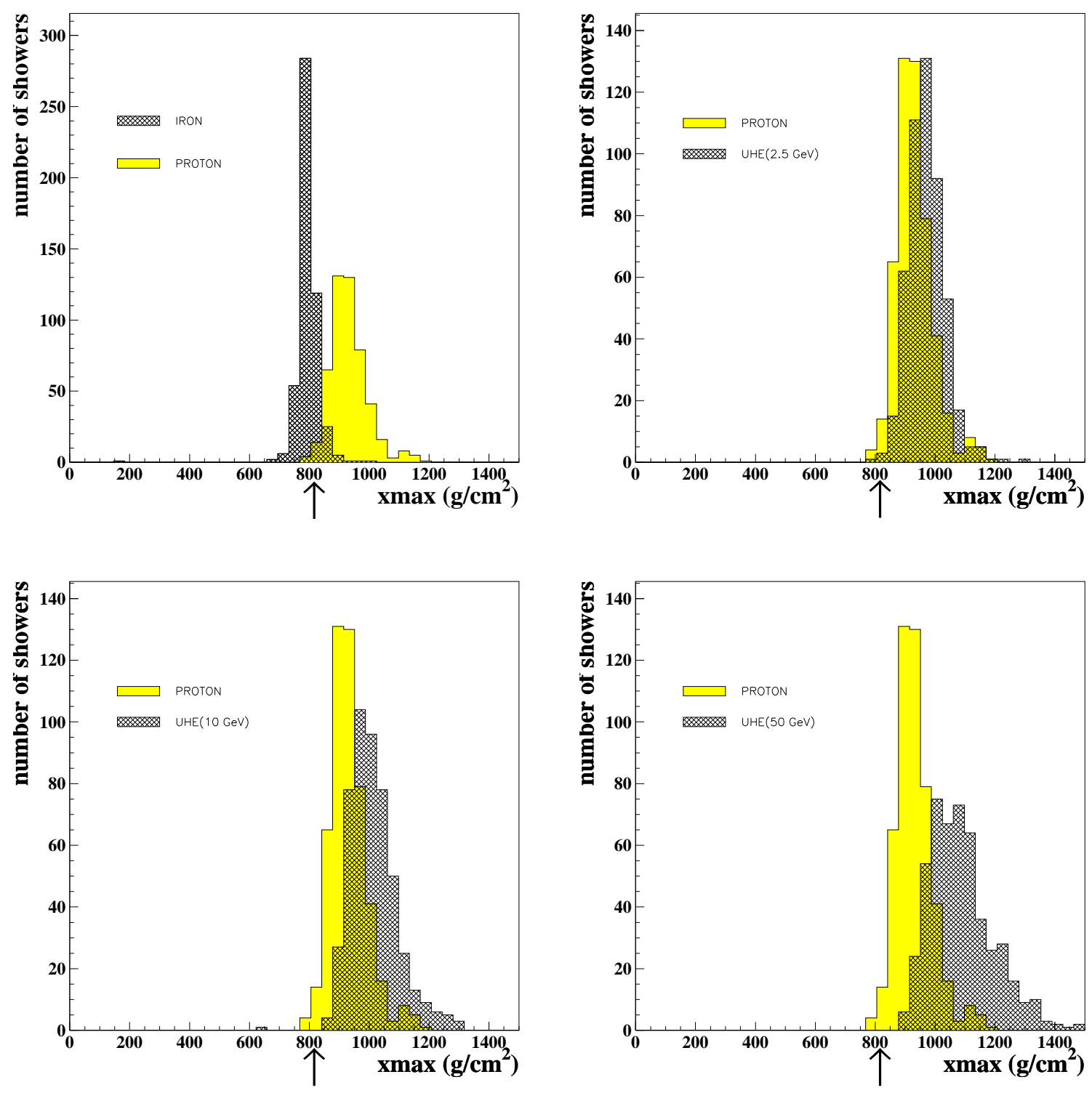

Figure 2: $X_{\text {MAX }}$ distribution for $320 \mathrm{EeV}$ showers. All plots are compared with the proton distribution: a) iron; b) $2.5 \mathrm{GeV}$ uhecron; c) $10 \mathrm{GeV}$ uhecron; d) $50 \mathrm{GeV}$ uhecron. The distributions are based on 500 showers for each case. The arrows indicate the value of $X_{M A X}$ for the Fly's Eye event, $815_{-53}^{+60}$. 


\subsection{Uhecron showers compared to observation}

From the $X_{M A X}$ distributions shown in Fig. 2 one can estimate the compatibility between these simulated showers and the Fly's Eye event. The energy of the observed event [7] is $320_{-94}^{+92} \mathrm{EeV}$, the $X_{M A X}$ is $815_{-53}^{+60} \mathrm{~g} \mathrm{~cm}^{-2}$, and the zenith angle is $43.9_{-1.3}^{+1.8}$ (the uncertainty is a combination of statistical and systematic effects). We use these values for the primary energy and the zenith angle in our simulations.

We compare our results with this Fly's Eye event because it is the only one with energy above the GZK cutoff detected with the atmospheric fluorescence technique. This allows a comparison of $X_{M A X}$ and the longitudinal distribution of our simulations with the observed event.

One can get a qualitative understanding why there will be a mass limit for the uhecron by examining Fig. 2. The distribution for a $2.5 \mathrm{GeV}$ uhecron is not very different from the proton distribution $\square$. But as the uhecron mass increases, the distribution becomes distinguishable from the proton distribution. The trend is that as the mass increases, $\left\langle X_{M A X}\right\rangle$ increases, as does the variance of the distribution.

The $320 \mathrm{EeV}$ event observed by Fly's Eye had $X_{M A X}$ of $815 \mathrm{~g} \mathrm{~cm}^{-2}$. This value is quite low compared to the mean of the distributions for large uhecron mass and therefore the observed event is unlikely to come from the probability distribution for large uhecron masses. The fact that the width of the uhecron's $X_{M A X}$ distribution increases with mass complicates this simple picture, but one can still estimate the maximum uhecron mass consistent with observation, keeping in mind the fact that there is but one event.

We will assume that the observed event was generated by a uhecron of unknown mass. The probability that the event resulted from a uhecron of mass $M_{i}$ is proportional to the probability for a uhecron of mass $M_{i}$ to generate the observed event (Bayes' rule

\footnotetext{
${ }^{10}$ Nor have we included sufficient refinement in the modeling for such a small difference to be significant.
} 
Table 1: The relative probability that a uhecron of mass $M_{i}$ produces an event with the indicated value of $X_{M A X}$.

\begin{tabular}{ccc}
\hline \hline Energy & $P\left(815 \mathrm{~g} \mathrm{~cm}^{-2} ; M_{i}\right)$ & $\frac{P\left(875 \mathrm{~g} \mathrm{~cm}^{-2} ; M_{i}\right)}{P\left(875 \mathrm{~g} \mathrm{~cm}^{-2} ; 2.5 \mathrm{GeV}\right)}$ \\
\cline { 2 - 3 }$P\left(815 \mathrm{~g} \mathrm{~cm}^{-2} ; 2.5 \mathrm{GeV}\right)$ & 1.00 \\
$2.5 \mathrm{GeV}$ & 1.00 & 0.39 \\
$5 \mathrm{GeV}$ & 0.23 & 0.33 \\
$10 \mathrm{GeV}$ & 0.24 & 0.21 \\
$20 \mathrm{GeV}$ & 0.17 & 0.09 \\
$40 \mathrm{GeV}$ & 0.08 & 0.12 \\
$50 \mathrm{GeV}$ & 0.11 & 0.06 \\
$100 \mathrm{GeV}$ & 0.04 & \\
\hline \hline
\end{tabular}

assuming equal priors). The probability distributions in Fig. 目 can be very well fit by a Gaussian plus an exponential tail. Let us refer to the normalized $X_{M A X}$ probability distribution for a uhecron of mass $M_{i}$ as $P\left(X_{M A X} ; M_{i}\right)$. The value of $P\left(815 \mathrm{~g} \mathrm{~cm}^{-2} ; M_{i}\right)$ is proportional to the probability that the observed event was drawn from $P\left(X_{M A X} ; M_{i}\right)$. We then compare the probabilities for various choices for the uhecron mass.

We arrive at a limit on the mass of the uhecron by demanding that $P\left(815 \mathrm{~g} \mathrm{~cm}^{-2} ; M_{i}\right)$ is at least $10 \%$ of its peak value. Since $P\left(X_{M A X} ; M_{i}\right)$ varies only slowly between $M_{i}=m_{p}$ and $2.5 \mathrm{GeV}$, we conservatively use $P\left(815 \mathrm{~g} \mathrm{~cm}^{-2} ; 2.5 \mathrm{GeV}\right)$ as our reference value for determining the uhecron mass limit 1 . In Table 1 we show the ratio $P\left(815 \mathrm{~g} \mathrm{~cm}^{-2} ; M_{i}\right) / P\left(815 \mathrm{~g} \mathrm{~cm}^{-2} ; 2.5 \mathrm{GeV}\right)$ for several values of $M_{i}$. One sees that for $M_{i}>40$ to $50 \mathrm{GeV}$ the probability is less than $10 \%$ of its value at $M_{i}=2.5 \mathrm{GeV}$. We therefore take this value as the largest uhecron mass compatible with observation. ${ }^{2}$ However, it must be noted that because large-mass uhecrons have broader distributions, $P\left(815 \mathrm{~g} \mathrm{~cm}^{-2} ; M_{i}\right)$ does not decrease as rapidly with $M_{i}$ as one might expect just on the

\footnotetext{
${ }^{11}$ By this procedure, we remove our sensitivity to shower-modeling inadequacies which may produce a shift between the predicted and observed $X_{M A X}$ distributions at lower energies.

${ }^{12}$ We also used $P\left(875 \mathrm{~g} \mathrm{~cm}^{-2} ; M_{i}\right)$, where $875 \mathrm{~g} \mathrm{~cm}^{-2}$ is the $1 \sigma$ upper limit of the observed event, and found that our limit does not change significantly.
} 
basis of the increase of $\left\langle X_{M A X}\right\rangle$. For instance, $P\left(815 \mathrm{~g} \mathrm{~cm}^{-2} ; 100 \mathrm{GeV}\right)$ is only about half as large as $P\left(815 \mathrm{~g} \mathrm{~cm}^{-2} ; 50 \mathrm{GeV}\right)$. The moral is that more data is needed, but just on the basis of one event, a uhecron of mass larger than 40 to $50 \mathrm{GeV}$ seems very unlikely.

\subsection{Achievable mass limit with additional data}

Now we turn to the question of how one might improve the limit on the uhecron mass with the observation of many more events. This discussion is meant to be just an indication of what might be obtained.

The most distinctive feature of the $X_{M A X}$ distribution for uhecron showers is the prominence of the tail for large $X_{M A X}$. As the mass increases, the tail becomes more pronounced. If sufficient events are observed to form the $X_{M A X}$ probability distribution function, then one can test for the size of the tail.

We proceed as follows:

1. For the proton and for various mass uhecrons, we fit the probability distribution functions of Fig. 2 to a Gaussian plus an exponential tail for large $X_{M A X}$. (The distributions are very well fit by this form.)

2. We generate a synthetic data set of $N$ events from the probability distribution function for a uhecron of mass $M$.

3. We fit the $N$ events generated in this way with the proton probability distribution function generated in step 1 . If it has a reasonable $\chi^{2}$, then we proceed, if not, we say that the data set generated in step 2 is incompatible with arising from proton-induced showers.

4. We subject data sets with a reasonable $\chi^{2}$ to another test by computing the number of events in the exponential tail and comparing it to the number of events in the Gaussian part of the distribution. 
5. If this ratio is the same (within $\pm 30 \%$ ) of that expected from the proton distribution, we say that the generated events are consistent with arising from the proton distribution function.

6. Then we repeat steps 2 through 5 many times. Some data sets of $N$ events will be consistent with a proton distribution, and some will not be consistent. We compute the percent of data sets that are consistent with the proton. If a large percentage of synthetic data sets are consistent, then we are unlikely on the basis of $N$ events to be able to differentiate between a uhecron of mass $M$ and a proton. If, on the other hand, only a small percentage of the synthetic data sets are consistent with a proton distribution, then we are very likely to be able to discriminate between a uhecron of mass $M$ and a proton on the basis of $N$ events.

7. We repeat the procedure for different $M$ and $N$.

The result of this procedure is shown in Fig. 3 . The trends in Fig. 3 are easy to understand. For a data set consisting of a given number of events, it is less likely for a large-mass uhecron to be consistent with a proton distribution. For a given uhecron mass, as the number of events increases, it becomes easier to differentiate a proton from a uhecron. The apparent rise at 50 events for a $10 \mathrm{GeV}$ uhecron is a statistical fluctuation.

We derive a "potential" mass limit from the fact that if the uhecron mass is larger than $10 \mathrm{GeV}$, there is better than a $95 \%$ probability one would be able to tell that the $X_{M A X}$ distribution was not generated by a proton from a data set with more than 30 events.

Again, we stress that this is just an illustrative calculation. For instance, we have not included any smearing of the $X_{M A X}$ probability distribution functions as a result of finite detector resolution. A full analysis of this issue must be left to the detector collaborations, but we can get a preliminary idea of its importance as follows. Let 
us assume that the experimentally determined $X_{M A X}$ is Gaussian distributed about the actual $X_{M A X}$, with width $\sigma_{E} \simeq 20 \mathrm{~g} \mathrm{~cm}^{-2}$. As can be seen from Fig. 2, the proton $X_{M A X}$ probability distribution function is also reasonably well fit by a normal distribution, due to the stochastic nature of particle production in hadronic showers. The width of the intrinsic $X_{M A X}$ spreading is rather large, $\sigma_{I} \simeq 50 \mathrm{~g} \mathrm{~cm}^{-2}$. Thus the reconstructed $X_{M A X}$

distribution will be Gaussian with width $\sigma=\sqrt{\sigma_{I}^{2}+\sigma_{E}^{2}}$. So long as $\sigma_{E}$ is smaller than $\sigma_{I}$, the experimentally measured width of the distribution would not be much different than shown in Fig. $⿴ 囗 ⿱ 一 一)$ using only the intrinsic smearing. A more serious issue would be if there are non-Gaussian tails in the detector resolution at large $X_{M A X}$. This would cause the observed proton distribution to resemble a uhecron distribution. A true limit on the uhecron mass will require a simulation including the detector response function, but unless there are large non-Gaussian tails in the detector response, our estimates should give a reasonable indication of what is achievable.

\subsection{The effect of the uhecron cross section}

We also investigated the dependence of the longitudinal profile on the cross section of the uhecron, as discussed in Section 2.3. In addition to using the same pion-nucleon cross section, we simulated showers where the cross section for the uhecron-nucleon interaction was $1 / 10$ of the pion-nucleon interaction. Seven samples were produced, for masses of $2.5,5,30,35,40,45$ and $50 \mathrm{GeV}$. The results of the cross section comparison are shown in Fig. 1 . As expected, the average $X_{M A X}$ is deeper for smaller cross section. Also the $X_{M A X}$ distribution is broader.

Analyzing these distributions to determine the maximum uhecron mass compatible with the observed shower, as in Section 3.2 above, shows that if the uhecron-nucleon cross section is lower than a standard hadronic cross section, the uhecron mass limit is even stronger. We find that for a cross section 1/10 the pion-nucleon cross section, the 


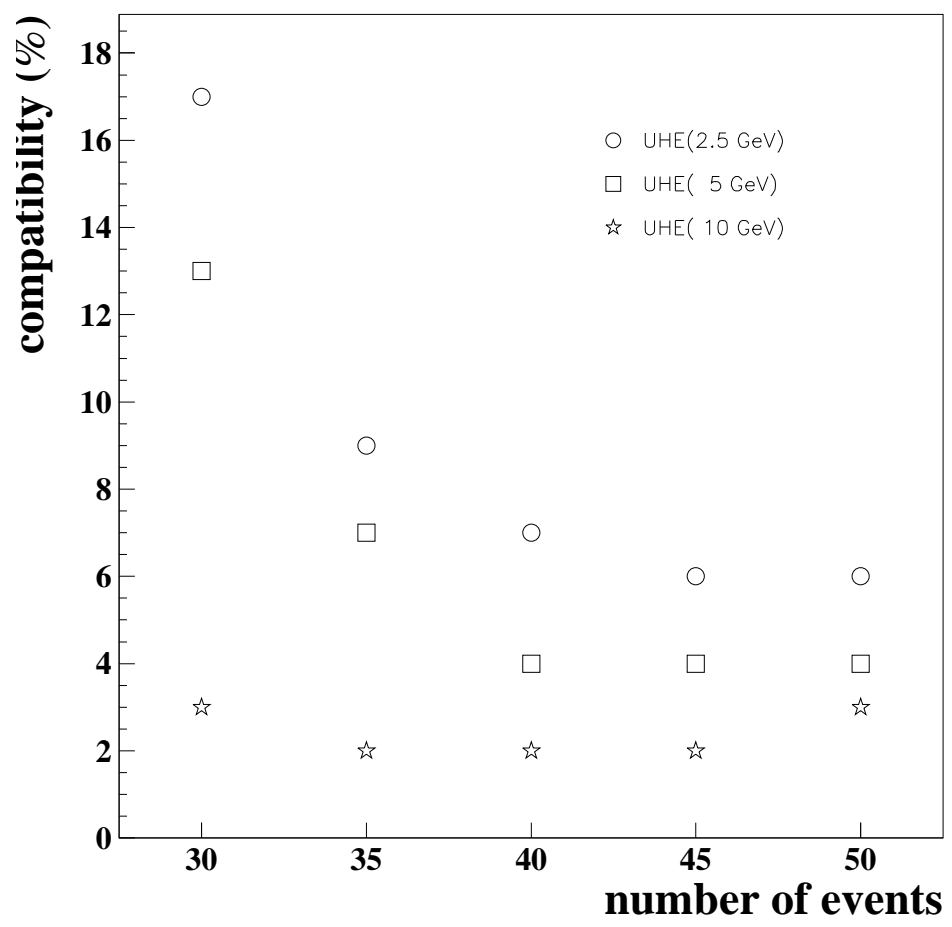

Figure 3: Percentage of the time a number of uhecron showers cannot be distinguished from a proton shower, for several different choices of the uhecron mass. For instance, given a data set with 40 showers, there is a $7 \%$ probability that the $2.5 \mathrm{GeV}$ uhecron $X_{M A X}$ distribution function will be well fit by the proton $X_{M A X}$ distribution function. For a $5 \mathrm{GeV}(10 \mathrm{GeV})$ uhecron, the probability is $4 \%(2 \%)$ if one has data from 40 showers.

uhecron mass must be lower than 35 to $40 \mathrm{GeV}^{\mathrm{T}}$.

\subsection{Other aspects of the shower development}

We show in Fig. 同 the energy distribution among the shower particles for a proton and a $50 \mathrm{GeV}$ uhecron. The first plot shows how the energy is distributed among nucleons, gammas, charged pions, and muons in a proton shower. The second plot shows the same

\footnotetext{
${ }^{13}$ For completeness, we also produced a sample for a $50 \mathrm{GeV}$ uhecron, assuming $\sigma_{U N}=2 \sigma_{\pi N}$, in order to assess the sensitivity to an increase in cross section. The change in the $X_{M A X}$ distribution is much smaller than in going from $\sigma_{U N}=\sigma_{\pi N}$ to $\sigma_{U N}=1 / 10 \sigma_{\pi N}$; it would have the effect of slightly weakening the limit on uhecron mass. Since we do not consider the larger cross section as physically well-motivated, we do not pursue this possibility further.
} 

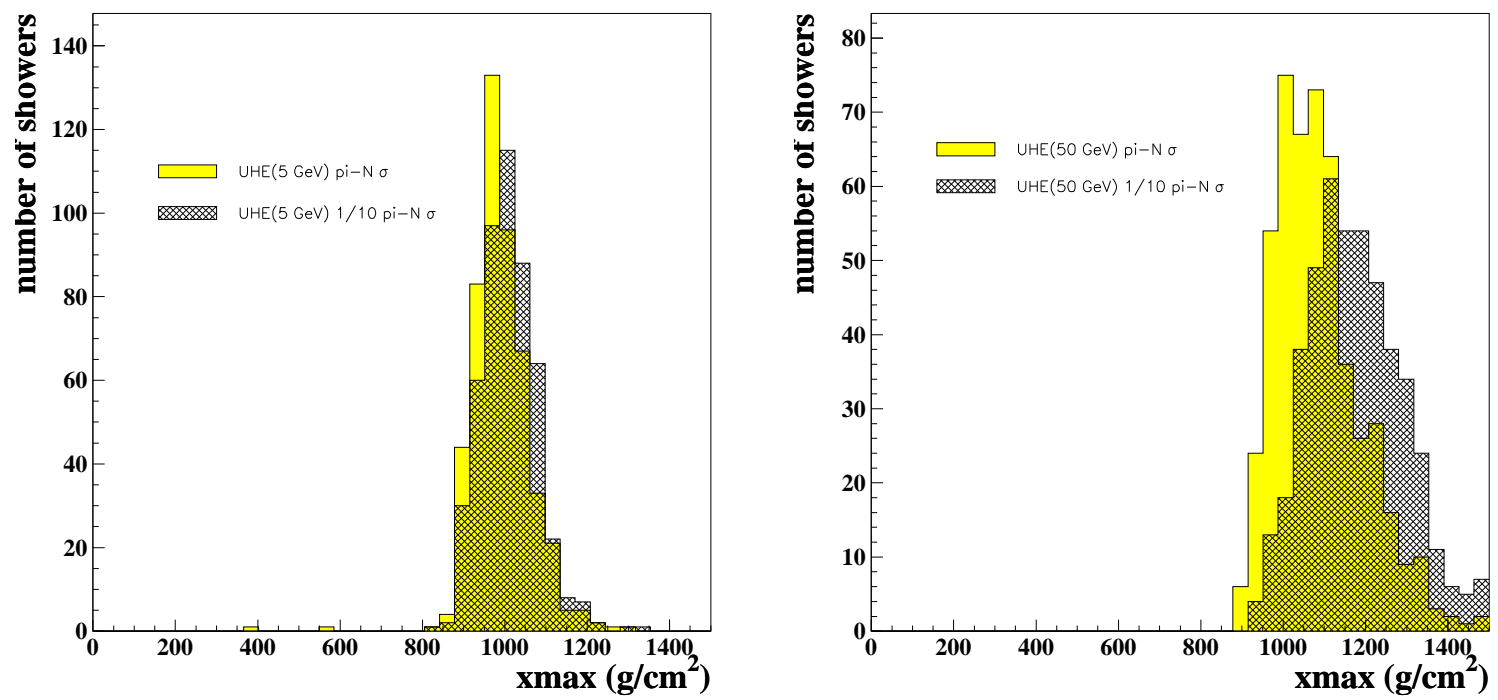

Figure 4: $X_{M A X}$ distributions for primaries with different cross sections. On the left, a $5 \mathrm{GeV}$ uhecron with $\sigma$ equal to the pi- $\mathrm{N}$ cross section and another with $1 / 10$ of this value are compared. On the right, the same for a $50 \mathrm{GeV}$ uhecron.

information for a shower initiated by a $50 \mathrm{GeV}$ uhecron, but showing the energy carried by the uhecron rather than the energy carried by nucleons. One sees that the uhecron carries a large fraction of the energy down to a considerable depth, whereas the energy in the proton shower is quickly distributed to other particles. We will return below to physical consequences of these distributions for using extensive air shower measurements to distinguish uhecron from proton primaries.

Finally, we also simulated showers for primaries with $100 \mathrm{EeV}$ energy. As there are no longitudinal distributions observed for events of this energy, we simply show the results in Table 2. The compatibility among uhecron and proton showers for this primary energy, can be deduced from the parameters in Table 2 . 

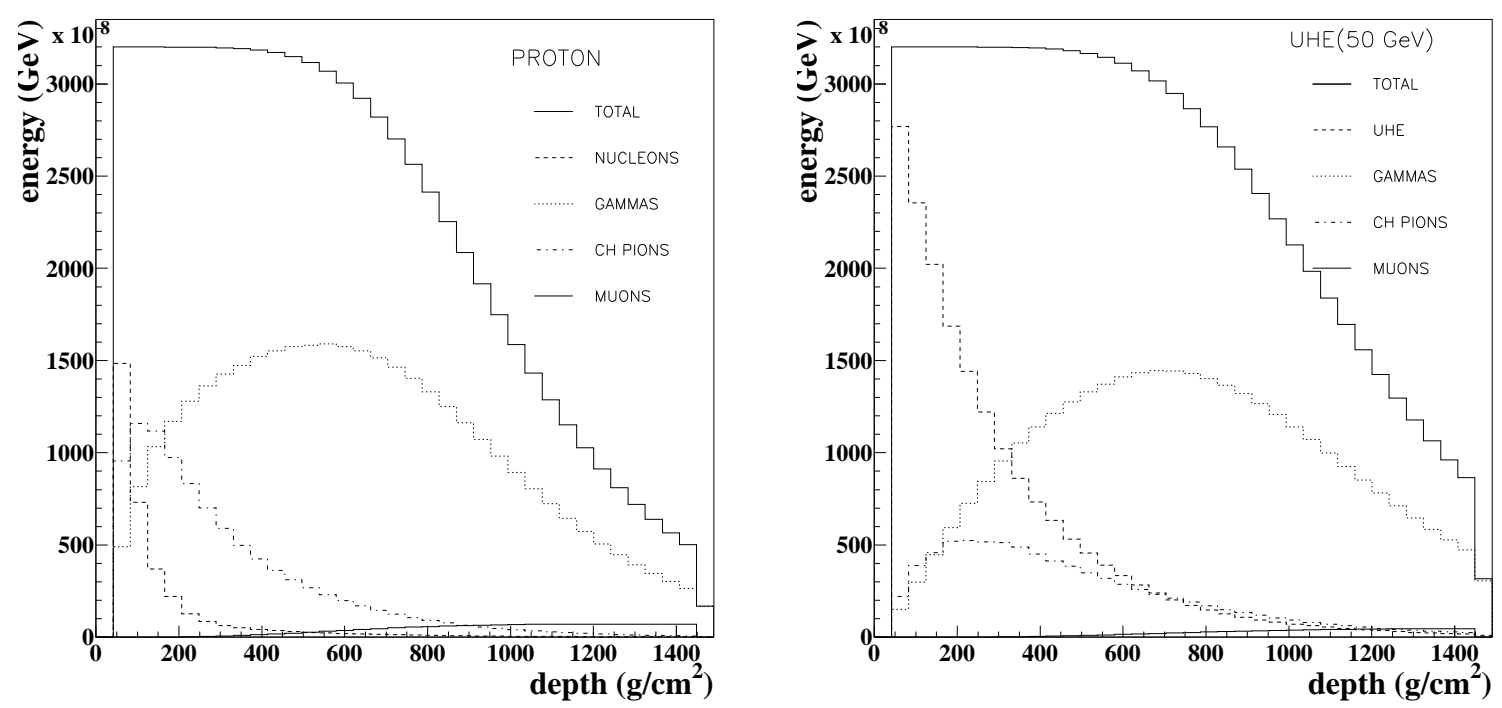

Figure 5: Distribution of energy among different constituents of the shower. Shown for the proton is the energy carried by all particles, by nucleons, gammas, charged pions and by muons. The same is shown for a $50 \mathrm{GeV}$ uhecron, with the exception that the energy carried by the uhecron is shown rather than the energy carried by nucleons.

\subsection{Ground level particle content}

In order to find a signature for the uhecron in an extensive air shower array detector, we studied the ratio between the muon $(\mathrm{mu})$ and the electromagnetic (em) density at ground level. To understand fully if this ratio can be used to distinguish a uhecron from other primaries, a complete analysis incorporating a model of the detector as well as thresholds and efficiencies would be needed. However our estimation can give an idea of

Table 2: The mean of the $X_{M A X}$ distribution and its variance $\sigma$ for various uhecrons of energy $100 \mathrm{EeV}$.

\begin{tabular}{ccc}
\hline \hline Primary & $\left\langle X_{M A X}\right\rangle\left(\mathrm{g} \mathrm{cm}^{-2}\right)$ & $\sigma\left(\mathrm{g} \mathrm{cm}^{-2}\right)$ \\
\hline Proton & 884 & 51 \\
uhecron $(5 \mathrm{GeV})$ & 949 & 59 \\
uhecron $(50 \mathrm{GeV})$ & 1031 & 79 \\
\hline \hline
\end{tabular}


the feasibility of this kind of signature.

The zenith angle for this analysis is chosen to be $30^{\circ}$ and ground level is taken to be $870 \mathrm{~g} \mathrm{~cm}^{-2}$. The distribution of the ratio $\mathrm{mu} / \mathrm{em}$ at 600 meters from the core is plotted in Fig. 6. The figure shows this ratio for a $5 \mathrm{GeV}$ uhecron and for a proton, and for a $50 \mathrm{GeV}$ uhecron and a proton.

From Fig. 6 one can see that the the mu/em ratio has a large dispersion. But the first plot shows there seems to be a qualitative difference between even a $5 \mathrm{GeV}$ uhecron and a proton: the proton mu/em ratio is larger than the $5 \mathrm{GeV}$ uhecron mu/em ratio. This difference can be understood by analyzing the muonic and electromagnetic longitudinal development, keeping in mind that the proton interaction with the atmosphere is very different from the uhecron interaction. At the top of the atmosphere, the particles produced by a proton shower are more energetic than the ones produced in a uhecron shower, since the light, strongly-interacting quanta carry a larger fraction of the initial energy in the former case. This can be seen from the plots of Fig. 5 .

The analysis of the muonic and electromagnetic longitudinal development shows that the difference in the number of muons between a proton and a uhecron shower is larger than the difference between the number of electromagnetic particles. This is due to the fact that the proton shower produces much more energetic pions in the top of the atmosphere. The charged pions therefore have enough time to interact, multiplying the number of pions, before decaying into muons. In the uhecron shower the charged pions at the top of the atmosphere are less energetic and will most likely decay directly into muons before interacting. This is not true for the electromagnetic component of the shower, which behaves in the same way in a proton and a uhecron shower, since a $\pi^{0}$ always decays rather than interacts.

The second plot in Fig. 6 is for a $50 \mathrm{GeV}$ uhecron. At first sight it is surprising that the uhecron $\mathrm{mu} / \mathrm{em}$ ratio is closer to the proton ratio than the $5 \mathrm{GeV}$ uhecron. This 
seems to be counter to the above explanation since the pions in the top of the atmosphere in the $50 \mathrm{GeV}$ shower should be even less energetic than the ones in a $5 \mathrm{GeV}$ shower. The effect, however, is due to an artifact of our choices of the ground level (made in order to be consistent with experimental sites) and the zenith angle for this analysis.

With the ground level depth at $870 \mathrm{~g} \mathrm{~cm}^{-2}$ and a $30^{\circ}$ zenith angle, the atmospheric depth is about $1005 \mathrm{~g} \mathrm{~cm}^{-2}$. This is enough for both a proton and a uhecron shower to have passed their maxima, while it is not enough for a $50 \mathrm{GeV}$ uhecron shower (see Fig. 11). Looking more closely at the muonic and electromagnetic longitudinal development, reveals that the muonic component of a $50 \mathrm{GeV}$ uhecron shower has not yet reached its maximum when arriving at ground level, whereas the electromagnetic component is almost at it's maximum at $1005 \mathrm{~g} \mathrm{~cm}^{-2}$. This is confirmed when one chooses a deeper ground level, where the $50 \mathrm{GeV} \mathrm{mu} / \mathrm{em}$ ratio is smaller than the $5 \mathrm{GeV}$ ratio.

An important point, evident from the difference between the fits to 300 and 150 proton showers in Fig. 6, is that very large statistics are essential to use mu/em as a tool for discriminating a uhecron from a proton. This, combined with the small shift in going from proton to uhecron, means that sensitivity to details of the shower modeling must be understood before the calculated difference can be trusted.

If both longitudinal shower development and the $\mathrm{mu} / \mathrm{em}$ ratio is available for a given event, correlated features may provide greater resolving power. As shown in a comparison between iron and proton showers [34], the mu/em ratio and the XMAX measurement are correlated and dependent on shower development parameters. Thus with large statistics, a multidimensional fit can be more sensitive than either alone. 

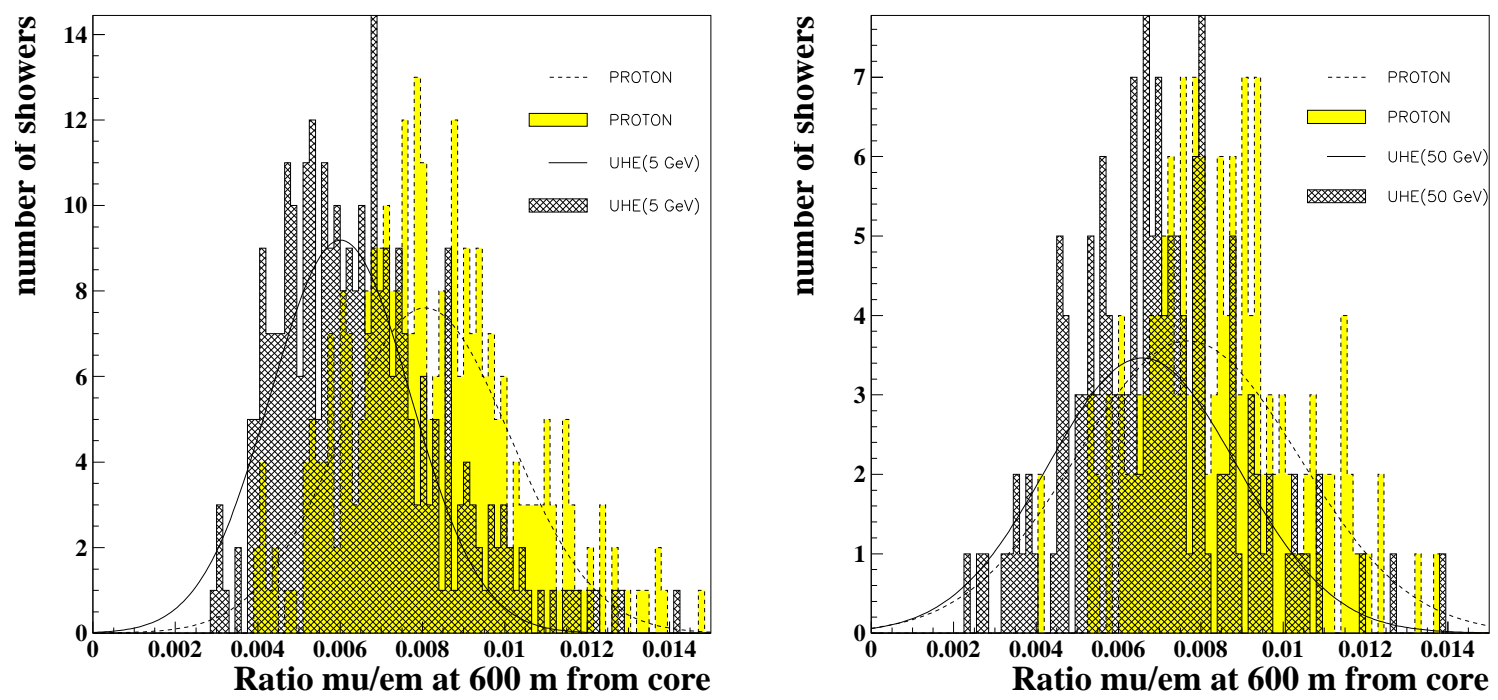

Figure 6: The muon to electromagnetic density ratio at ground level. The left figure is the result of 300 showers each for a $5 \mathrm{GeV}$ uhecron and a proton, while the right figure is 150 showers each for a $50 \mathrm{GeV}$ uhecron and a proton.

\section{Discussion and Conclusion}

We conclude from our simulations that a uhecron (a massive, long-lived and strongly interacting particle) that penetrates Earth's atmosphere can produce an air shower with characteristics qualitatively similar to those of a proton shower. We find an approximate upper limit of $50 \mathrm{GeV}$ on the mass of such a particle in order to have a shower compatible with the longitudinal development observed in the highest energy cosmic ray event [7]. If the cross section of the uhecron is lower than a "normal" hadronic cross section, this limit is even more stringent. For instance it drops to $40 \mathrm{GeV}$ for a cross section 1/10 of the pion-nucleon cross section.

We note that as the mass of the uhecron increases, the shower develops more slowly (larger average $X_{M A X}$ ) and the fluctuations in $X_{M A X}$ increase. With a large number of events whose longitudinal shower development is measured, these differences could 
be sufficient to distinguish uhecron from proton primaries. We estimate that about 30 events may be enough to discriminate between uhecron and proton, if the uhecron is more massive than $10 \mathrm{GeV}$. At lower uhecron mass the discrimination is difficult because the longitudinal development profiles become too similar. In this case the ratio of muon over electromagnetic density at large distances from the core could be helpful with very high statistics. We conjecture that having combined information on a single event, longitudinal development as well as lateral shower properties and the mu/em ratio, may give improved resolving power. Such combined information would be available if the event were observed simultaneously with both fluorescence and ground level detectors, as in the Pierre Auger project [34].

It is encouraging that approved and proposed experimental efforts [34, 35, 36] are likely to produce a substantial increase in the statistics available on ultrahigh energy cosmic rays. We have shown that the detailed shower properties can allow candidate new-particle explanations for the highest energy events to be ruled out or conceivably confirmed. Many possible new-particle explanations are already excluded. In particular, the highest energy cosmic ray event is very unlikely to have been produced by a new superheavy $(M \gtrsim 100 \mathrm{GeV})$ hadron containing a heavy colored particle or monopole.

We would like to thank Clem Pryke, Jim Cronin, Gustavo Burdman, Sergio Sciutto and Albert Stebbins for useful discussions. IA was supported in part by NSF Grant AST 94-20759 and the DOE through grant DE-FG02-90ER40606. The work of GRF was supported by NSF-PHY-94-23002. The work of EWK was supported at Fermilab by the Department of Energy and by NASA under number NAG5-7092.

\section{References}


[1] K. Greisen, Phys. Rev. Lett. 16, 748 (1966).

[2] G. T. Zatsepin and V. A. Kuźmin, Pis'ma Zh. Eksp. Teor. Fiz. 4, 114 (1966).

[3] M. A. Malkan and F. Stecker, Ap. J. 496, 13 (1998); F. Stecker, Phys. Rev. Lett. 80, 1816 (1998); F. Stecker and O.C. De Jager, astro-ph/9804196; L. N. Epele and E. Roulet, astro-ph/9808104; F. Stecker and M. Salamon, astro-ph/9808110.

[4] J. Linsley, Phys. Rev. Lett. 10, 146 (1963).

[5] G. Brooke et al., Proc. of 19th International Cosmic Ray Conference, La Jolla (1985).

[6] N. N. Efimov et al. Proc. Of International Workshop on Astrophysical Aspects of the Most Energetic Cosmic Rays, editors: M. Nagano and F. Takahara, World Scientific, 1990.

[7] D. J. Bird et al., Ap. J. 441, 144 (1995).

[8] N. Hayashida et al., Proc. of International Symp. on Extremely High Energy Cosmic Rays, Tokyo, Japan, Sept., 1996.

[9] J. W. Elbert and P. Sommers, Ap. J. 441, 151 (1995).

[10] G. R. Farrar and P. L. Biermann, astro-ph/9806242, to be published Phys. Rev. Lett.

[11] S. A. Colgate, private communication; P. Blasi and A. Olinto, astro-ph/9806264; G. Sigl, M. Lemoine and P. Biermann, astro-ph/9806283.

[12] C. T. Hill, D.Schramm and T. Walker, Phys. Rev. D 34, 1622 (1986); F. A. Aharonian, P. Bhattacharjee and D. N. Schramm, Phys. Rev. D 46, 4188 (1992); J. H. MacGibbon and R. H. Brandenberger, Phys. Rev. D 47, 2283 (1993). 
[13] E. Waxman, Ap. J. 452, L1 (1995).

[14] F. Halzen, R. A. Vázquez, T. Stanev and H. P. Vankov, Astropart. Phys. 3, 151 (1995).

[15] G. Burdman, F. Halzen and R. Gandhi, Phys. Lett. B 417, 107 (1998).

[16] T. J. Weiler, preprint hep-ph/9710431.

[17] G. R. Farrar, Phys. Rev. Lett. 76, 4111 (1996).

[18] D. J. H. Chung, G. R. Farrar and E. W. Kolb, Phys. Rev. D 57, 4606 (1998).

[19] S. Raby, Phys. Lett. B422, 158 (1998).

[20] R. N. Mohapatra and S. Nussinov, Phys. Rev. D 57, 1940 (1998).

[21] E. Nardi and E. Roulet, Phys. Lett. B 245, 105 (1990).

[22] T. W. Kephart and T. J. Weiler, Astropart. Phys. 4, 271 (1996); Nucl. Phys. Proc. Suppl. 51B, 218 (1996).

[23] C. O. Escobar and R. A. Vázquez, astro-ph/9709148.

[24] R. M. Baltrusaitis et al., Nucl. Instr. Meth. A240, 410 (1985).

[25] C.Pryke, private communication.

[26] T. K. Gaisser and A. M. Hillas, Proc. 15th Internat. Cosmic Ray Conf, Plovdiv, 8, $353(1977)$.

[27] A. Watson, Proceedings of the 1997 Durban Symposium on High Energy Cosmic Rays.

[28] S. J. Sciutto, GAP-98-005 (Pierre Auger project technical note) (1998). 
[29] A. M. Hillas, Proc. of 19th International Cosmic Ray Conference, La Jolla, 1985.

[30] M. T. Dova and S. J. Sciutto, GAP-97-053 (Pierre Auger project technical note) (1997).

[31] R. S. Fletcher, T. K. Gaisser, P. Lipari and T. Stanev, Phys. Rev. D50, 5710 (1994).

[32] N. Isgur and M. B. Wise, Phys. Lett. B232, 113 (1989); ibid B237, 527 (1990). For a review see M. Neubert, Phys. Rep. 245, 259 (1994).

[33] R. M. Barnett et al., Phys. Rev. D54 (1996).

[34] Auger Collaboration, The Pierre Auger Project Design Report, 1996.

[35] M. Al-Seady et al., Proc. International Symposium on Extremely High Energy Cosmic Rays: Astrophysics and Future Observatories, edited by M. Nagano, Tokyo (1996).

[36] N. Hayashida et al., ibid. 